\title{
A Parametric Study of Solute Redistribution During Transient Liquid Phase Diffusion Bonding Process
}

\author{
Tien-Chien Jen, ${ }^{1}$ Yuning Jiao, ${ }^{1}$ and Thomas Hwang ${ }^{2}$ \\ ${ }^{1}$ Mechanical Engineering Department, University of Wisconsin-Milwaukee, Milwaukee, Wisconsin, USA; \\ ${ }^{2}$ Combustion Engineering, GE Power Systems, Schenectady, New York, USA
}

\begin{abstract}
A parametric study is performed to investigate the solute redistribution during the transient liquid phase (TLP) diffusion bonding process. The macroscopic solute diffusion in the liquid and the solid phase, as well as the solid transformation to the liquid due to solute macrosegregation, are considered in this study. The effects of the following parameters are considered: ratio of solute diffusivity in liquid and solid state alloy $\left(\xi=D_{l} / D_{s}\right)$, holding temperatures $(\theta)$, a combined parameter related to solidus and liquidus slopes in the phase diagram $(\varphi)$, and the re-melting and re-solidification time $(\tau)$. The thickness of the pure liquid zone and the mushy zone of the TLP diffusion bonding process are demonstrated with respect to the above-mentioned parameters. It is shown numerically that the holding time, the holding temperature, and solute diffusivity ratio influence the solute distribution strongly, which in turn influences the liquid zone and mushy zone thickness significantly. It is concluded that for the TLP diffusion bonding process, the optimal technique parameters are high holding temperature, long holding time, and a large liquidus and solidus temperature slope ratio $\left(m_{l} / m_{s}\right)$ of the interlayer material.
\end{abstract}

Keywords Parametric study, Solute redistribution, TLP process, Diffusion bonding

\section{INTRODUCTION}

Among many bonding techniques, the transient liquid phase (TLP) process is unique in the sense of producing similar or even the same microstructure within the bonding interface. It is well known that in commercial welding technology, the

Received in final form on 23 July 2001.

Address correspondence to Tien-Chien Jen, Mechanical Engineering Department, University of Wisconsin-Milwaukee, Milwaukee, WI 53211.E-mail: jen@cae.uwm.edu microstructure and crystal growing direction are changed due to non-uniform heating and cooling processes (Milton Lima et al., 1998). This usually gives rise to lower bonding strength, in particular in high temperature environments. Owing to the solid and liquid state diffusion, film molten zone and explicit crystal growth during the TLP process, single crystal growth is possible (Gale and Abdo, 1999; Bradley, 1988; Zheng et al., 1987; and Ruan et al., 1996). The motivation of the present study is trying to develop an enabling technology to bond two single crystal bulk materials with the maximum possible bonding strength using the TLP process technique. Note that this is particularly important to applications such as turbine blade systems in the aerospace industry (Gale and Guan, 1999).

The principle of the transient liquid phase technique is to bond two crystals by using an interlayer alloy. The melting temperature of the interlayer alloy should be less than the substrate (master) alloy. The isothermal heat treatment temperature is determined to be above $30-50^{\circ} \mathrm{C}$ of the melting point of interlayer materials. After isothermal heat treatment, the interlayer is remelted and the liquid fills fully of the two interfaces of the single crystals. The composition of bonds will reach equilibrium composition and move along the tie line. Thus, the solidification temperature increases and the volume of liquid decreases. The growth is similar to that of laser re-melted metals, epitaxial growth based on the base materials. Finally, after sufficient isothermal heat treatment, the liquids disappear and the two single crystals bond into one single crystal without the formation of re-crystallized zone at the bonds if the two single crystals have the same orientation.

Transient liquid phase bonding has been developed to join superalloys susceptible to hot cracking (Bradley, 1988). It is very important to design a suitable interlayer alloy that does not contain any deleterious phases and has a melting point lower than that of the base metal (Gale and Wallach, 1991; Gale and Wallach, 1990a; Gale et al., 1992; and Gale and Wallach, 1990b). At present, the interlayer alloys that are generally used for superalloys are of the Ni-Cr-Si-B system. However, the addition of $\mathrm{Si}$ and $\mathrm{B}$ should be avoided because these elements are harmful 
impurity elements in single-crystal superalloys. Hafnium (Hf) is a beneficial element for improving the intermediate temperature creep properties and strengthening the $\gamma^{\prime}$ phase (Gale and Guan, 1999). Thus, an interlayer containing Hf was developed for bonding DD3 Ni-based single crystals and the $\gamma / \gamma^{\prime}$ two-phase structure had been obtained in the bonding layer (Guan and Gale, 1999). However, the interlayer containing Hf was developed for first-generation Ni-based superalloys that are Re-free superalloys. Until now, there was no investigation of interlayer materials for TLP bonding Re-containing Ni-based superalloys. Therefore, it is necessary to develop a new interlayer with high properties to maintain the strength of the bond and keep the strength to be equivalent to that of the base alloy.

So far, almost all the earlier works concentrated on the microstructure research. Zheng et al. (1987) improved the TLP process to bond two nickel-based single crystals without a second phase existing in the bonds. They solved this problem by developing an interlayer containing $\mathrm{Hf}$ without $\mathrm{B}$ and $\mathrm{Si}$. They found the melting temperature of $\mathrm{Ni5Hf}$ is $1130-1160^{\circ} \mathrm{C}$ and the maximum solubility of $\mathrm{Hf}$ in $\gamma^{\prime}$ can reach 7at\%. Ohashi et al. (1996) investigated the relationship between the disorientation angle at the bonding interface and joint strength. Gale and Guan (1999) presented and investigation of transient liquid phase bonding of single crystal NiAl to a conventional polycrystalline nickel-based superalloy MM247. They proposed a wide gap TLP bonding process in their study. In wide gap TLP bonding, a composite interlayer consisting of a liquidforming matrix, plus a (nominally) non-melting constituent, is employed.

Accurate control of the crystal growth of bonding two bulk single crystals by the TLP is crucial to improve the product quality, in particular in the aerospace industry, such as turbine blade systems. Computer simulation in re-melting and solidification is useful to predict the microstructure, crystal orientation, and mechanical properties during the TLP. However, so far, numerical simulation has rarely been used in the development of the bonding of single crystal alloys, especially in the TLP. The primary objective of the present study is to develop a mathematical model of the solute diffusion that can be used for TLP. The macroscopic solute diffusion in the liquid and the solid, as well as the solid transformation to liquid due to solute macrosegregation, are considered in this study. The effects of holding temperatures, the diffusivity ratio of liquid and solid alloys on the holding time, the thickness of the pure liquid zone, and the mush zone on the TLP bonding process are investigated.

\section{THEORETICAL ANALYSIS}

Consider two master single crystal alloys to be joined with an interlayer in between as shown in Fig. 1. It is assumed that the thermal properties and mass diffusivities are constant, and the entire system is exposed to a constant holding temperature with

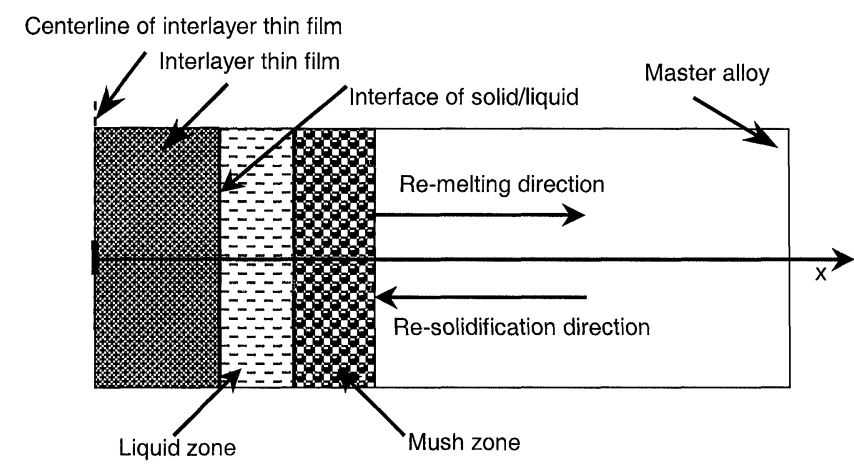

FIGURE 1

Schematic representation of the physical domain.

the system instantaneously reaching this temperature. Typically, the interlayer is about $30-50^{\circ}$ lower than the master alloys' melting temperature. Thus, it is important to choose the appropriate holding temperature that should be between the melting temperature of the interlayer and the master alloy. As shown in Fig. 1, a liquid interlayer initially is formed in between two master alloys. As time increases, due to the mass diffusion into the master alloy, the melting temperature decreases near the interlayer. Thus, a re-melting layer was formed and the interface moves into the master alloy. Note that the thickness of this re-melting layer is critical in determining the bonding strength of the TLP process. Also, a mushy zone is generated due to solute redistribution in the master alloy; this causes the coexistence of a liquid and solid phase. Note that the formation of the mushy zone may be detrimental to single crystal growth and consequently decreases the strength of the bonding layer strength.

\section{Mathematical Formulation}

The model, based on Fick's law, including the effect of solid remelting, is used in the present study. By adding the volume conservation equations within the solid and liquid phase, the following macroscopic conservation equation for the solute concentration is obtained ( $\mathrm{Ni}$ and Beckermann, 1991):

$$
\frac{\partial\left(\rho_{l, s} C_{l, s}\right)}{\partial t}+\frac{\partial\left(\rho_{l, s} C_{l, s} U_{l}\right)}{\partial x}=-\frac{\partial j_{c}}{\partial x}
$$

where $t, \rho_{l, s}, C_{l, s}, U_{l}$, and $j_{c}$ are the time, liquid or solid density, liquid or solid solute concentration, superficial liquid velocity, and solute diffusive flux, respectively. Assuming the solid phase is stationary and the dispersion flux is negligible, the diffusive solute flux can be modeled as follows:

$$
j_{c}=-g_{l, s} \rho_{l, s} D_{l, s} \frac{\partial C_{l, s}}{\partial x},
$$

where $g_{l, s}$ is the volume fraction of liquid or solid and $D_{l, s}$ is the effective solute diffusivity in the liquid or solid. When the 
mushy zone is small, we have $g_{l, s}$ equals one. Thus, Equation (2) can be modeled as follows:

$$
j_{c}=-\rho_{l, s} D_{l, s} \frac{\partial C_{l, s}}{\partial x}
$$

By assuming the thermodynamic equilibrium at the solidliquid interface and a uniform concentration locally in the liquid, the liquid concentration can be related to the temperature by the liquidus of the phase diagram as follows:

$$
C_{l}=\left(T-T_{M}\right) / m_{l},
$$

where, $T_{M}$ is the melting point of the pure solvent and $m_{l}$ is the slope of the liquidus.

The interlayer and master alloy are assumed to be initially at a uniform solute concentration:

$$
\begin{gathered}
C_{I}=C_{I 0}, \quad \text { at } \quad 0<x<L / 2, t=0, \\
C_{M}=C_{M 0}, \quad \text { at } \quad x>L / 2, t=0 .
\end{gathered}
$$

The boundary conditions in the TLP process is as follows:

Solute concentration for far away from the interlayer is assumed to remain at the initial concentration $C_{M 0}$ :

$$
C_{M}=C_{M 0}, \text { at } \quad x=\infty .
$$

At the interlayer centerline, the concentration flux can be obtained due to the symmetric distribution of the solute concentration:

$$
\left.\frac{\partial C_{I}}{\partial x}\right|_{x=0}=0
$$

The equations listed above can be non-dimensionalized with the interlayer thickness $L$ as the characteristic length, $L^{2} / D_{L I}$ as the characteristic time, and $C_{0}$ as the characteristic solute concentration. The following dimensionless distance $X$, time $\tau$, concentration $\phi$, and holding temperature $\theta$ can be defined:

$$
X=\frac{x}{L}, \quad \tau=\frac{t D_{L I}}{L^{2}}, \quad \phi=\frac{C}{C_{I 0}}, \quad \theta=\frac{T}{m_{l} C_{0}} .
$$

All the equations listed above can then be non-dimensionalized as follows:

For the liquid region in the interlayer (LI),

$$
\frac{\partial \phi_{L I}}{\partial \tau}=\frac{\partial^{2} \phi_{L I}}{\partial X^{2}}
$$

For the solid region in the interlayer (SI),

$$
\frac{\partial \phi_{S I}}{\partial \tau}=\frac{\partial}{\partial X}\left(\frac{D_{S I}}{D_{L I}} \frac{\partial \phi_{S I}}{\partial X}\right)
$$

For the liquid region in the master alloy (LM),

$$
\frac{\partial \phi_{L M}}{\partial \tau}=\frac{\partial}{\partial X}\left(\frac{D_{L M}}{D_{L I}} \frac{\partial \phi_{L M}}{\partial X}\right)
$$

and for the solid region in the master alloy (SM),

$$
\frac{\partial \phi_{S M}}{\partial \tau}=\frac{\partial}{\partial X}\left(\frac{D_{S M}}{D_{L I}} \frac{\partial \phi_{S M}}{\partial X}\right)
$$

The dimensionless form of Equation (4) can be obtained,

$$
\phi_{(S, L) M}=\theta-\theta_{m}
$$

The non-dimensional initial conditions for the solute concentration in the interlayer and master alloy can be given, respectively,

$$
\begin{aligned}
\phi_{I} & =1, \quad \text { at } \quad 0<X<1 / 2, \tau=0, \\
\phi_{M} & =0, \quad \text { at } \quad X>1 / 2, \tau=0 .
\end{aligned}
$$

The non-dimensional boundary conditions are as follows,

$$
\begin{gathered}
\phi_{M}=\phi_{M 0}, \quad \text { at } \quad X=\infty, \\
\left.\frac{\partial \phi_{M}}{\partial X}\right|_{X=0}=0 .
\end{gathered}
$$

It can be seen from the dimensional analysis that four sets of dimensionless parameters are generated: $D_{S I} / D_{L I}, D_{L M} / D_{L I}$, $D_{S M} / D_{L I}$, and $\theta$. The first three parameters are the mass diffusivity ratio between the solid solute and the liquid solute in the master alloy and interlayer. The last parameter is dependent on holding temperature and the material physical properties. In general, the effective mass diffusivities of the liquid solute (i.e., $D_{L I}$ and $D_{L M}$ ) can be assumed to be identical, and same assumption can be made for the effective mass diffusivities of the solid solute (i.e., $D_{S I}$ and $D_{S M}$ ). With these assumptions, the parameters are now reduced to only two: $D_{S I} / D_{L I}$ and $\theta$. Here we redefine the first parameter as

$$
\xi=\frac{D_{l}}{D_{s}}
$$

where $D_{l}$ and $D_{s}$ denote the mass diffusivity of the liquid and the solid, respectively. Physically, this parameter denotes the relative mass diffusion penetration capabilities of the liquid solute versus the solid solute. The larger the ratio, the faster the solute concentration penetrates further into the mass alloy. The second parameter is the dimensionless holding temperature. In this study, we will limit our investigation to the effect of the holding temperatures $(\theta)$, the relative mass diffusion penetration capability $\left(\xi=D_{l} / D_{s}\right)$, and the holding time $(\tau)$ to the solute redistribution in the interlayer and master alloy for three different types of aluminum alloys ( $\mathrm{Al}-\mathrm{Cu}(4.5 \% \mathrm{wt}), \mathrm{Al}-\mathrm{Mg}$ 
(4.5\% wt), and Al-Si (2.0\% wt) alloys), which are equivalent to $\xi=36,46$, and 56 , respectively.

\section{Numerical Method and Grid Convergence Tests}

The governing equations are solved numerically by the explicit finite volume method (Patankar, 1980). In calculation, we assume that the solute diffusion coefficient in solid or liquid is the same; $D_{L I}=D_{L M}, D_{S I}=D_{S M}$. The computational domain is chosen in such way that it is large enough (i.e., from $3000 \mu \mathrm{m}$ to $20,000 \mu \mathrm{m}$ ) to ensure the penetration depth is small enough in comparison to the total computation domain. For representative purposes, only the Al-Cu alloy phase diagram is shown in Fig. 2. Note that in order to calculate the liquidus and solidus temperature for the solute, linear regressions for both curves are used:

$$
\begin{array}{cl}
\text { Liquidus: } & T_{L}=T_{A l}-m_{l} \times C_{A l} \\
\text { Solidus: } & T_{S}=T_{A l}-m_{S} \times C_{A l}
\end{array}
$$

where $T_{L}, T_{S}$, and $T_{A l}$ denote liquidus, solidus, and pure aluminum melting temperatures, respectively. $m_{l}$ and $m_{s}$ are the slopes of the liquidus and solidus curves in the phase diagram, and $C_{A l}$ is the solute concentration (in wt \%). Note that the $T_{A l}, m_{l}, m_{s}, D_{l}$, and $D_{s}$ for different alloys can be obtained from Table III.

To ensure the grid size and time step are small enough for the convergence of the solution, a detailed grid convergence tests have been performed as shown in Table I. It can be seen from the table that when the grid size changes from $0.025 \mu \mathrm{m}$ to $0.5 \mu \mathrm{m}$ and the time steps vary from $0.05 \mathrm{sec}$ to $1 \mathrm{sec}$, the numerical error being less than $3.7 \%$. Thus, a grid size of $\Delta x=$ $0.25 \mu \mathrm{m}$ and a time step of $\Delta \mathrm{t}=1 \mathrm{sec}$ are used throughout the present study to ensure the grid independence. This model is further verified by comparing the numerical results presented by Havard and Asbjorn (1997) for the solute redistribution of

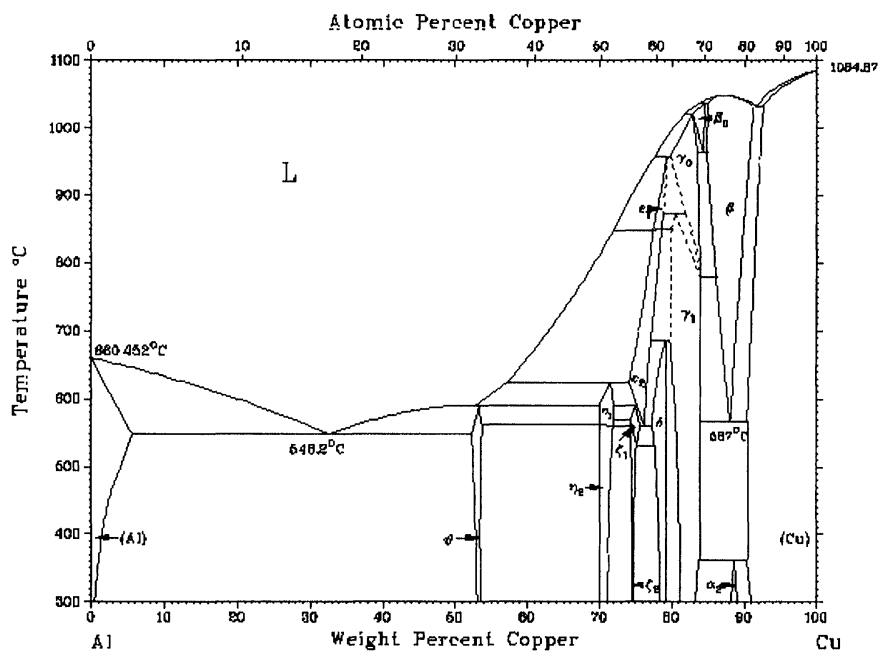

FIGURE 2

Al-Cu phase diagrams.
TABLE I

Grid size and time step convergence tests

\begin{tabular}{lllllll}
\hline $\mathrm{t},(\mathrm{sec})$ & 1 & 1 & 1 & 0.05 & 0.1 & 0.5 \\
$\Delta \mathrm{x},(\mu \mathrm{m})$ & 0.5 & 0.1 & 0.05 & 0.5 & 0.5 & 0.5 \\
Maximum error of & $3.7 \%$ & $2.2 \%$ & $2.3 \%$ & $1.3 \%$ & $2.4 \%$ & $1.6 \%$ \\
$\quad$ solute concentration \\
$\quad$
\end{tabular}

$\mathrm{Al}-\mathrm{Cu}(4.5 \mathrm{wt} \%)$ alloy in the unidirectional solidification without the effect of re-melting. The agreements between the present model and Havard and Asbjorn's results were found to be very good (not shown). With this agreement and the grid convergence tests shown before, we are confident that this numerical model is valid to further compute the solute redistribution during the TLP.

\section{RESULTS AND DISCUSSION}

In order to quantify the effect of macro-scale solute diffusion on the solid/liquid interface movement, re-melting layer, and mush zone thickness, as well as the solidification time, the solute concentration distributions in the interlayer and master alloy are investigated. In this study, a one-dimensional model has been modified to account for the macroscopic solute diffusion in the liquid and the solid, as well as for the solid transformation to the liquid due to the solute macrosegregation. This model is then applied to A1-4.5 pct $\mathrm{Cu}, \mathrm{Al}-4.5$ pet $\mathrm{Mg}$, and $\mathrm{Al}-2.0$ pet $\mathrm{Si}$ interlayer alloy ( $\xi=35,46$, and 56 , respectively), and a pure Al master material, which is held at different non-dimensional temperatures $(\theta)$ corresponding to $40.720,41.035,41.351$, and 41.667 , respectively. A parameter, $\varphi=\left(\frac{D_{l}}{D_{s}}\right) *\left(\frac{m_{l}}{m_{s}}\right)$, is defined in the present study to introduce the combined effect of the mass diffusion ratio of the liquid and solid phase, and the slope ratio between liquidus and solidus lines in the phase diagram. The corresponding values of $\varphi$ of different materials are shown in Table III.

\section{The Solute Redistribution from the Centerline of Interface}

Figure 3 demonstrates the solute spatial concentration distribution for two kinds of alloys, $\xi=35$ and $\xi=56$ at four different non-dimensional holding temperatures $(\theta=40.720$, $41.035,41.351$, and 41.667) and three different non-dimensional holding times ( $\tau=12600,50400$, and 126000). It can be seen from the figure that the solute concentration decreases progressively as the holding temperature increases. This is because the higher holding temperature leads to a larger liquid state solute region, which has higher effective solute mass diffusivity (i.e., $D_{l} \gg D_{s}$ ). This effect is more pronounced at longer times (i.e., longer than $\tau=126000$ ) as shown in the figure. The solute concentration decreases as the time increases in the interlayer region where very significant mass penetration occurs, and in contrast, for master alloys, the solute concentration increases as time increases. This causes the re-melting of the master alloy in 
TABLE II

The dimensionless solute concentration intervals for different alloy in different phase regions at various dimensionless holding temperatures

\begin{tabular}{clcccc}
\hline \multirow{2}{*}{$\begin{array}{c}\text { Dimensionless } \\
\text { concentration, } \phi\end{array}$} & \multicolumn{4}{c}{ Dimensionless temperature, $\theta$} & \multicolumn{2}{c}{0.0290} \\
\cline { 3 - 6 }$\xi=35$ & Liquid zone & $\phi>0.9756$ & 0.6600 & 0.3442 & $\phi>0.0284$ \\
& Mush zone & $0.1836<\phi<0.9756$ & $0.1242<\phi<0.6600$ & $0.0647<\phi<0.3440$ & $0.0053<\phi<0.0171$ \\
& Solid zone & $\phi<0.1836$ & $\phi<0.1242$ & $\phi<0.0647$ & $\phi<0.0053$ \\
$\xi=46$ & Liquid zone & $\phi>0.5862$ & $\phi>0.3964$ & $\phi>0.2069$ & $\phi>0.0171$ \\
& Mush zone & $0.2533<\phi<0.5862$ & $0.1713<\phi<0.3964$ & $0.2069<\phi<0.0893$ & $0.0073<\phi<0.0171$ \\
& Solid zone & $\phi<0.2533$ & $\phi<0.1713$ & $\phi<0.0893$ & $\phi<0.0073$ \\
$\xi 56$ & Liquid zone & $\phi>0.9945$ & $\phi>0.6725$ & $\phi>0.3510$ & $\phi>0.0290$ \\
& Mush zone & $0.1955<\phi<0.9945$ & $0.1320<\phi<0.6725$ & $0.0690<\phi<0.3510$ & $0.0055<\phi<0.0290$ \\
& Solid zone & $\phi<0.1955$ & $\phi<0.1320$ & $\phi<0.0690$ & $\phi<0.0055$ \\
\hline
\end{tabular}

the short time when the solute concentration in the master alloy exceeds the melting point concentration at the holding temperature. Similarly, re-solidification occurs at longer times when the solute concentration is below the solidus curve. All these phenomena will be discussed in greater detail later. In addition, the solute concentration distribution is related to diffusivity and the phase diagram (see Fig. 2).

The solute concentration gradient at the moving liquid/solid interface is tracked and shown in Fig. 4. It is worth pointing out that the concentration gradient at the solid/liquid interface is the driving force for the re-melting and re-solidification of the master alloy in the TLP. It can be seen from this figure that with increasing holding times, the solute concentration gradient decreases quickly, and becomes constant at larger holding times. With the increase of holding temperatures, the solute concentration gradient decreases. This means at greater holding temperatures, the concentration gradient becomes smaller. This reveals that at lower holding temperatures the solute mass diffusion is stronger. Note that this does not mean that the concentration is lower for low holding temperatures since a greater holding temperature has a larger liquid region, which has a higher effective solute diffusion coefficient (see Fig. 3). An interesting phenomenon observed in this figure is that for the curve of the holding temperature at $\theta=40.720$ for alloy $\varphi=19.888$, the solute concentration gradient decreases significantly from beginning and moves close to zero after $\tau=16000$. This is because the re- solidification phenomena occur when the solute concentration falls below to its solidus concentration value (see Fig. 3). Table II shows the solute concentration intervals for interlayer alloy in different phase regions at various holding temperatures. On the other hand, as the parameter $\varphi=\left(\frac{D_{l}}{D_{s}}\right) *\left(\frac{m_{l}}{m_{s}}\right)$ increases, the solute concentration gradient increases. It is because with the increase of solute diffusivity in the liquid state (or the decrease of solute diffusivity in the solid state), solute atoms will diffuse toward to liquid/solid interface quickly. However, because of the relatively small solute diffusivity in the solid state, these solute atoms will concentrate at the liquid side around the liquid/solid interface, and form the macrosegreation. This increases the solute concentration gradient near the solid/liquid interface. The increase of the liquidus slope (or the decrease of the solidius slope) will cause the decrease of the mush zone thickness (or the solidification point will move toward the high concentration region). This causes the solute concentration to increase quickly at the liquid/solid interface.

\section{The Effect of Solute Diffusion on Solid/Liquid Interface Movement}

As mentioned above, the solute diffusion causes the melting temperature change in the interlayer and master alloy (see Table I and Eqs. (20) and (21)). Because of the decrease of the solute concentration in the interlayer, its melting temperature increases; while at master alloy, its melting temperature

TABLE III

Some parameters related to the phase diagrams and the alloys

\begin{tabular}{lcccccc}
\hline & $\begin{array}{c}m_{l} \\
\left({ }^{\circ} \mathrm{C} / \mathrm{wt} \%\right)\end{array}$ & $\begin{array}{c}m_{s} \\
\left({ }^{\circ} \mathrm{C} / \mathrm{wt} \%\right)\end{array}$ & $\begin{array}{c}D_{l} \\
\left(\mathrm{~mm}^{2} / \mathrm{s}\right)\end{array}$ & $\begin{array}{c}D_{s} \\
\left(\mathrm{~mm}^{2} / \mathrm{s}\right)\end{array}$ & $\begin{array}{c}T_{A l} \\
\left({ }^{\circ} \mathrm{C}\right)\end{array}$ & $\varphi$ \\
\hline $\mathrm{Al}-\mathrm{Cu}$ & 3.520 & 18.71 & 0.0035 & 0.0001 & 660.452 & 6.5850 \\
$\mathrm{Al}-\mathrm{Mg}$ & 5.858 & 13.55 & 0.0046 & 0.0001 & 660.452 & 19.888 \\
$\mathrm{Al}-\mathrm{Si}$ & 7.770 & 39.55 & 0.0056 & 0.0001 & 660.452 & 11.002 \\
\hline
\end{tabular}




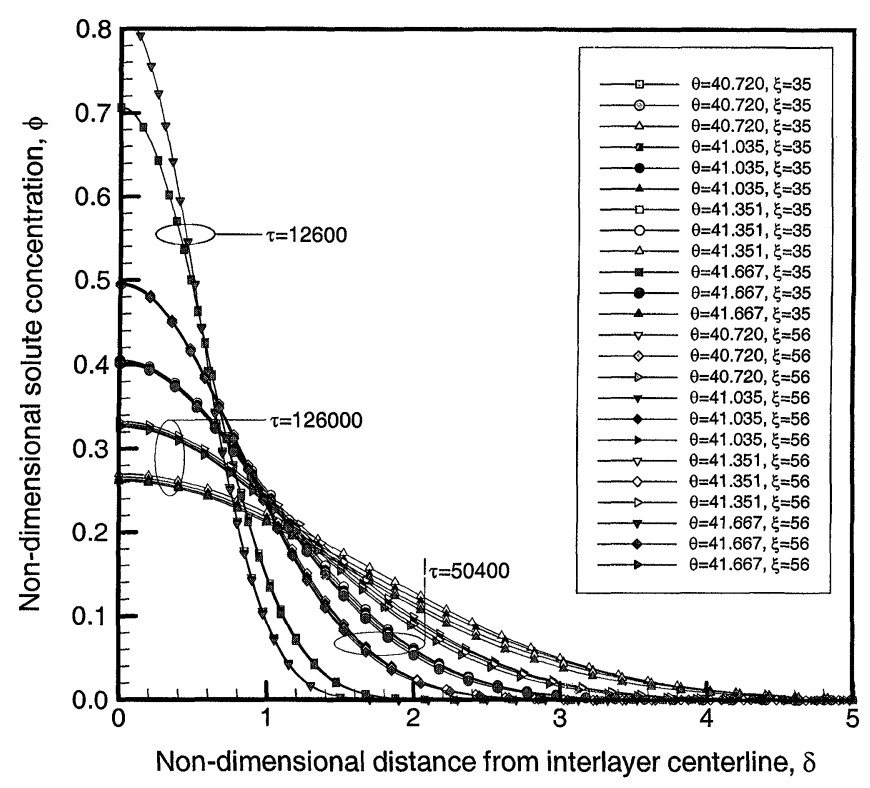

FIGURE 3

The solute spatial non-dimensional concentration distribution for two alloys at different non-dimensional holding temperatures and holding times.

decreases due to increases in solute concentration. Thus, the master alloy may be molten where is contiguous to the interlayer depending on the holding temperature applied. If the holding temperature is greater than the melting temperature adjacent to the interlayer, the master alloy will be molten; this results in the solid/liquid interface movement. Figure 5 indicates the solid/liquid interface movement at different holding

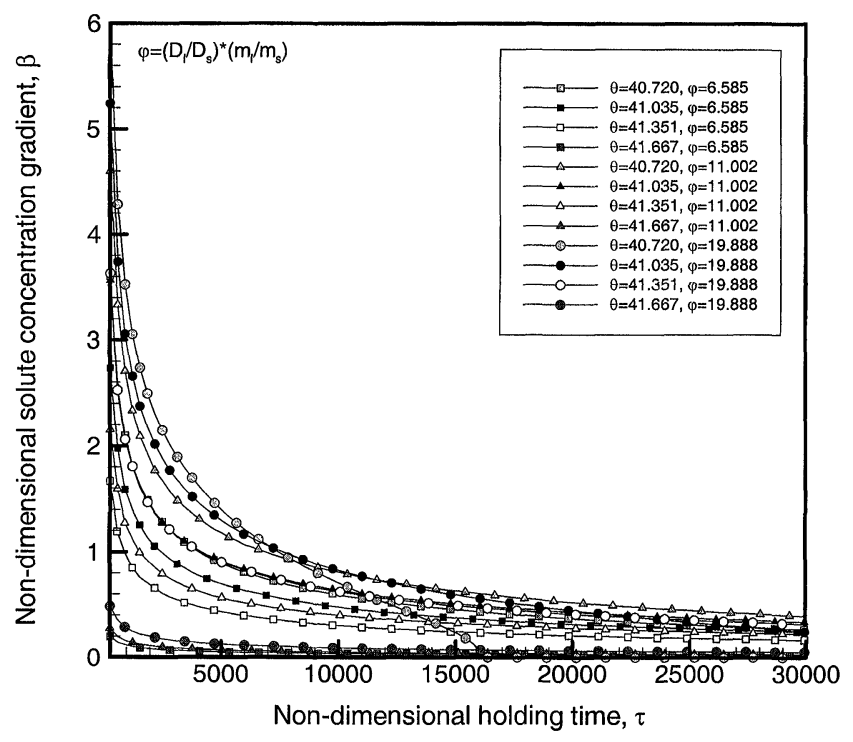

FIGURE 4

The non-dimensional solute concentration gradient at the moving liquid/solid interface. temperatures and holding times for two different interlayer alloys (i.e., $\xi=35$ and 46 ). It can be seen that with the increase of the holding temperature, the solid/liquid interface moves further into the master alloy as holding time increases until it reaches a maximum re-melting layer thickness. After this maximum thickness is reached, the re-melting layer thickness starts to decrease, so the re-solidification process begins. This will continue until all the material solidifies (for both interlayer and master alloy, shown in Fig. 5 as arrows pointed). This phenomenon is caused by the solute concentration re-distribution as the mass diffusion of solute penetrates further into the master alloy. Note that the solute concentration in the interlayer decreases as the holding time increases, and the solute concentration of the master alloy in the region near the interlayer increases in the early stages (Jen and Jiao, 2001). Thus, it causes the re-melting layer to grow. However, the solute concentration decreases as time progresses, and, eventually, the driving mass concentration in the interlayer falls below the liquidus concentration. Thus, at that point, the re-melting layer starts to solidify. These can be seen clearly in the figure, for example, for the case with $\xi=$ $46, \theta=40.720, \xi=46, \theta=41.035$; and $\xi=35, \theta=40.720$. In these curves, the re-melting layer starts to grow from the beginning until the maximum re-melting layer thickness is reached. After this holding time, it starts to solidify until the complete re-melting layer disappears. For the same alloy, the increase of the holding temperature causes the maximum thickness of re-melting layer to increase, which requires much greater holding time. For example, for the case of a holding temperature $\theta=40.720$ (low holding temperature), the time required for reaching the maximum re-melting layer is much less than the case of a high holding temperature $(\theta=41.035)$.

The graph shown in Fig. 6 is a partial enlargement of Fig. 5 in the dotted line area. It depicts the effect of the holding temperature and time on the thickness of the mush zone in the master alloy material. Note that the mush zone thickness is critical for single crystal growth in the master alloy. In general, the mush zone is detrimental to the formation of a single crystal alloy, and thus should be either avoided or eliminated in some way to optimize the strength of the bonding. The arrows in the figure show the merging point of the liquid zone and the mush zone. That means that beyond this point the thickness of the liquid zone and the mush zone is equal, which indicates the pure liquid zone disappears. It can be seen from this figure that there is a pure liquid zone in the interlayer region at the beginning of the holding time and the thickness of the mush zone is equal to zero. With the increase of the holding time, the thickness of the liquid zone and the mush zone increase progressively, then the thickness of the pure liquid zone decreases until these two curves merge with each other. Furthermore, with the increase of the $\xi$ value, the required holding time to the merging point increases, but the thickness at the merging point decreases. That means a pure liquid zone will remain for a long time for a large value of $\xi$, which is beneficial for diffusion bonding. The reason for this phenomenon occurs is that the increase of the solute 


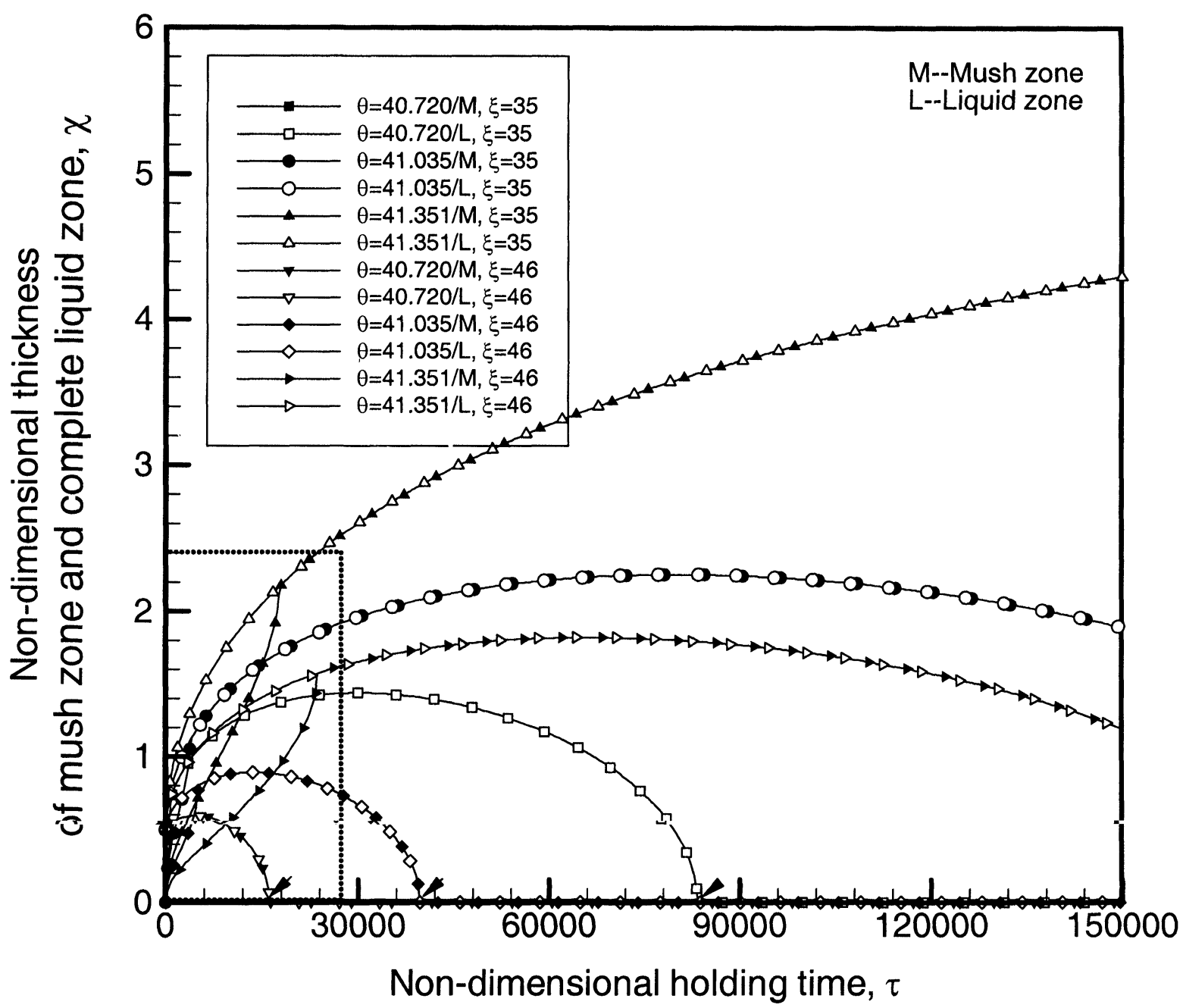

FIGURE 5

The effect of the non-dimensional holding time on the non-dimensional thickness of mush zone and liquid zone for different alloys at different holding temperature. (Arrows show the solidification time.)

diffusivity ratio (by decreasing $D_{s}$ or increasing $D_{l}$ ) will decrease the penetration depth of solute atoms in the solid state. Thus, it takes a longer holding time for the solid phase concentration to reach the solute concentration of a two-phase zone, thereby decreasing the re-melting layer thickness at the merging point. In addition, for the same interlayer alloy, the holding time and the thickness of the mush zone at the merging point increases with increasing holding temperature. This is because with the increased holding temperature, the liquid state region increases (see Fig. 2 and Table II). For the same reason, it needs a much longer time for the solid phase concentration to reach the solute concentration of a two-phase zone. From the design point for single crystal bonding, the holding temperature should be as close to the melting temperature of the master alloy as possible, since the pure liquid phase layer is the largest. This is favorable, since a single crystal can be re-grown from the single crystal master alloy.

\section{The Re-melting and Re-solidification Time}

Figure 7 shows the maximum re-melting thickness and the holding time to achieve this thickness with respect to $m_{s} / m_{l}$ (i.e., for three different kinds of interlayer alloys). It can be seen from the figure that the maximum re-melting layer thickness has a linear relationship with the ratio of the slope of the solidus and liquidus lines and the layer thickness increases with this ratio. The time required to achieve this thickness also increases with this ratio. Furthermore, the increase of holding temperature increases the maximum thickness of the re-melting layer, as well as the holding time required to achieve this thickness. The increase in solidus line slope means that the solute concentration required for solid phase at a given holding temperature decreases and this means the region in which solid and liquid phase coexists increases.

Note that it is desirable to have a larger re-melting layer (stronger bonding) and shorter holding time (less energy 


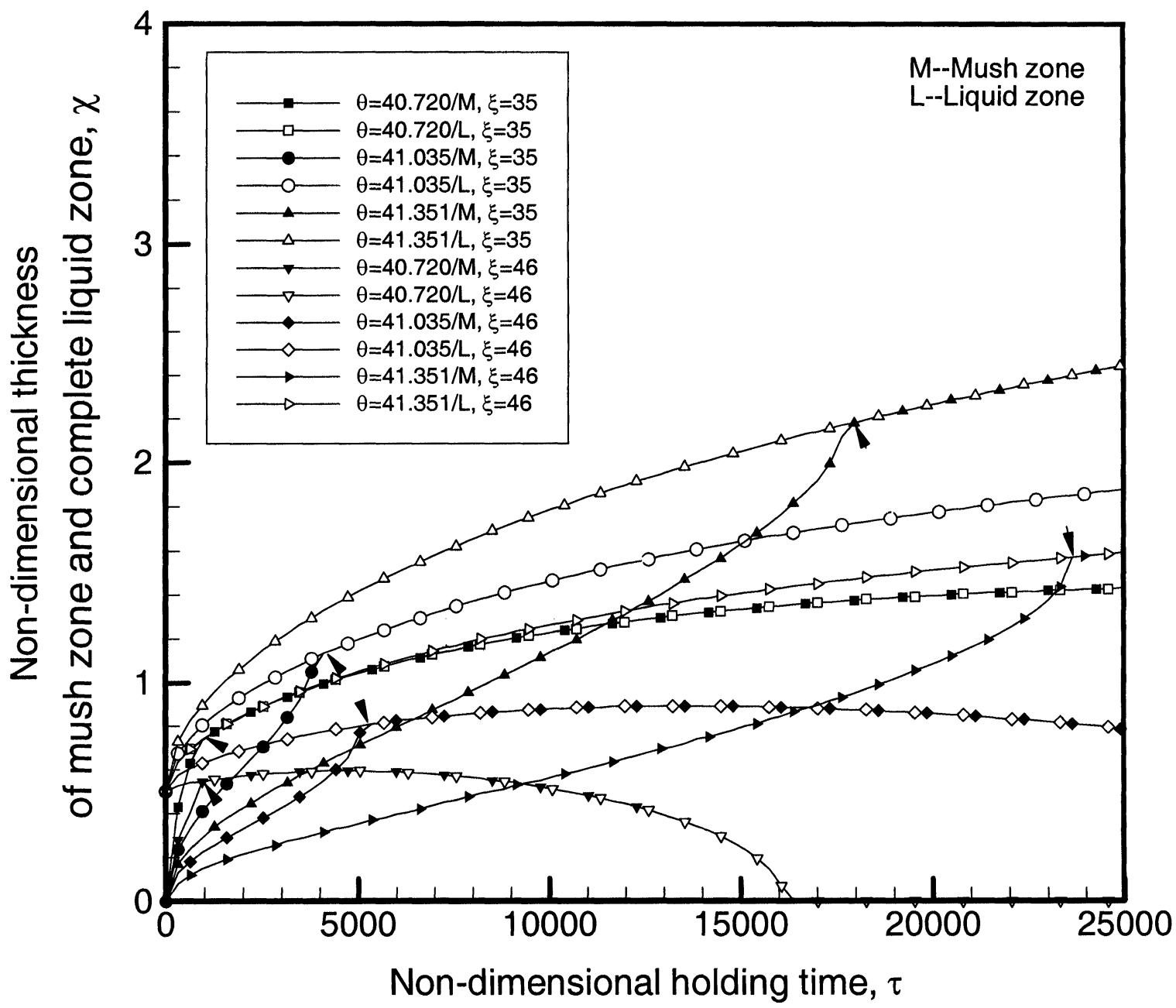

FIGURE 6

The effect of the non-dimensional holding time on the non-dimensional thickness of mush zone and liquid zone for different alloys at different holding temperature. (Arrows show the pure liquid zone disappears.) (Enlargement of Figure 5)

consumption). However, as can be seen from the figure, it is virtually impossible to achieve both goals. Instead, a judgment must be made to choose preferred conditions to obtain the most economical solution.

\section{CONCLUSIONS}

A parametric study is performed to investigate the solute redistribution during the transient liquid phase (TLP) diffusion bonding process. The macroscopic solute diffusion in the liquid and the solid phase, as well as for the solid transformation to the liquid due to solute macrosegregation, are considered in this study. The effects of the following parameters are considered: ratio of solute diffusivity in liquid and solid state alloy $(\xi=$ $\left.D_{l} / D_{s}\right)$, holding temperatures $(\theta)$, a combined parameter related to phase diagram $(\varphi)$, and the re-melting and re-solidification time $(\tau)$. The thickness of the pure liquid zone and the mush zone of the TLP diffusion bonding process are demonstrated with respect to the above-mentioned parameters. Several major conclusions from the present study can be drawn as follows:

- The solute concentration decreases as the holding temperature increases. Near the interlayer, the solute concentration decreases as holding time increases, while away from the interlayer, the solute concentration increases with holding time.

- The solute concentration gradient decreases as the holding temperature increases at the solid/liquid interface. This reveals that the re-solidification process is slower for higher holding temperatures, which leads to much longer TLP process times. Also, the solute concentration gradient increases as the liquid phase solute diffusivity and the slope of liquidus increase.

- In general, the thickness of the mush zone increases with the holding time. At lower holding temperatures, the mush zone occupies a great portion of the 


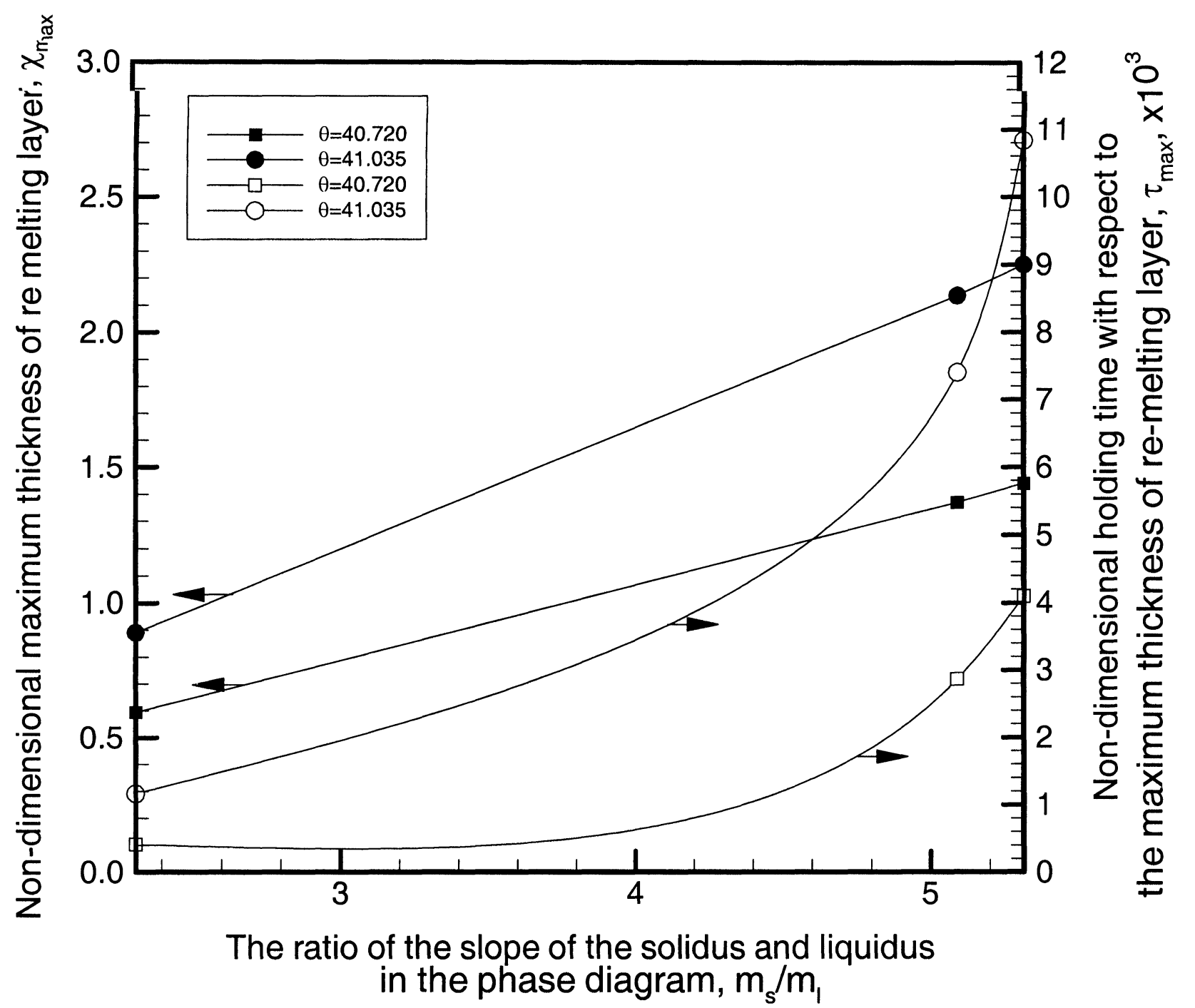

FIGURE 7

The effect of the ratio of the slope of the solidus and liquidus on non-dimensional maximum thickness of re-melting layer and with respect to the holding time.

re-melting layer. When the holding temperature approaches the melting point of master alloy, the pure liquid layer becomes the dominant layer in the re-melting layer.

- With increased holding temperature, the solid/ liquid interface moves further into the master alloy with respect to time until it reaches a maximum re-melting layer thickness. After this maximum thickness is reached, the re-melting layer thickness starts to decrease, when the re-solidification process begins.

- The maximum re-melting layer thickness increases linearly with the ratio of the slope of the solidus and liquidus $\left(m_{l} / m_{s}\right)$. The time required to achieve this thickness also increases with the ratio. Furthermore, the increase of holding temperature increases the max- imum thickness of the re-melting layer and the required holding time to achieve this thickness.

- The optimal parameters for the TLP bonding process are high holding temperatures, long holding times, and large $\left(m_{l} / m_{s}\right)$ interlayer materials.

\section{NOMENCLATURE}

$C$ solute concentration

$D$ effective solute diffusivity

$g \quad$ volume fraction of liquid or solid

$j_{c} \quad$ solute diffusive flux

$L \quad$ interlayer thickness

$m_{l} \quad$ slope of the liquidus

$m_{s} \quad$ slope of the soludius

$t$ time

$T$ holding temperature 
$T_{L} \quad$ liquidus temperature

$T_{M} \quad$ melting point of the pure solvent

$T_{S} \quad$ solidus temperature

$U_{l} \quad$ superficial liquid velocity

$x \quad$ spatial coordinate perpendicular to the solid/liquid interface

$X$ dimensionless $\mathrm{x}$-coordinate

\section{Greek Symbols}

$\delta \quad$ dimensionless thickness

$\phi \quad$ dimensionless concentration

$\varphi \quad$ dimensionless parameter, $\left(\frac{D_{l}}{D_{s}}\right) *\left(\frac{m_{l}}{m_{s}}\right)$

$\theta$ dimensionless holding temperature

$\rho$ density

$\tau$ dimensionless time

$\xi$ ratio of solute diffusivity in liquid and solid state alloy, $D_{l} / D_{s}$

\section{Subscripts}

Al pure aluminum

I interlayer

I0 initial condition of interlayer

$l \quad$ liquid phase

$L I \quad$ interlayer in liquid state

$L M$ master alloy in liquid state

$M$ master alloy

$\mathrm{m}$ melting point of master alloy

MO initial condition of master alloy

$s \quad$ solid phase

SI interlayer in solid state

SM master alloy in solid state

\section{REFERENCES}

Bradley, E. F., 1998, In Superalloys-A Technical Guide, (ASM International, Metals Park, OH), pp. 22-28.

Gale, W. F., and Abdo, Z. A. M., 1999, Bulk Alloy Micro-structural Analogues for Transient Liquid Phase Bonds in the NiAl/Cu/Ni System, Metall. Mater. Trans. A, vol. 30A, no. 12, pp. 3111-3124.

Gale, W. F., and Guan, Y., 1999, Transient Liquid Phase Bonding of $\mathrm{NiAl}$ Intermetallic Compound to Nickel-Base Superalloys-A Microstructural Investigation, Proceedings of the Fifth International Conference on Trends in Welding Research, ASM International, pp. 663-668.
Gale, W. F., and Guan, Y., 1999, Wide-Gap Transient Liquid Phase Bonding of NiAl-Hf to a Nickel-Base Superalloy, Mater. Sci. Technol., vol. 15, pp. 464-467.

Gale, W. F., Totemeier, T. C., and King, J. E., 1992, Transformation of Aluminide Intermetallic Coatings on Single Crystal Nickel-Base Superalloys During Heat Treatment and Subsequent Exposure, Proceedings of Heat and Surface '92, Editor: I. Tamura, Japan Technical Information Service, pp. 483-486.

Gale, W. F., and Wallach, E. R., 1990a, Wetting of Nickel Alloys by Nickel Based Brazes, Mater. Sci. Technol., no. 6, pp. 170-175.

Gale, W. F., and Wallach, E. R., 1990b, Wetting Mechanisms in HighTemperature Brazing of Nickel-Based Alloys, Recent Trends in Welding Science and Technology, Editors: David S. A., and Vitek J. M., ASM, pp. 529-534.

Gale, W. F., and Wallach, E. R., 1991, Influence of Isothermal Solidification on Microstructural Development with Ni-Si-B Filler Metals, Mater. Sci. Technol., no. 7, pp. 1143-1148.

Guan, Y., and Gale, W. F., 1999, Transient Liquid Phase Bonding of a Hf-bearing Single-Crystal Nickel Aluminide to MM-247 Superalloy, Mater. Sci. Technol., vol. 15, pp. 207-212.

Havard, J. T., and Asbjorn, M., 1997, The Effect of Macroscopic Solute Diffusion in the Liquid upon Surface Macrosegregation, Metallurgical Transactions B, vol. 28B, pp. 665-669.

Jen, T. C., and Jiao, Y., 2001, Numerical Simulation of Solute Redistribution During Transient Liquid Phase Bonding Process for Al$\mathrm{Cu}$ Alloy, Numerical Heat Transfer-Part A: Applications, vol. 39, no. 2, pp. 123-138.

Milton Lima, S. F., Gilgien, P., and Kurz, W., 1998, Microstructure Selection in Laser Remelted Fe-C-Si Alloys, Z. Metallkd, vol. 89, no. 11, pp. 751-757.

Ni, J., and Beckermann, C., 1991, A Volume-Averaged Two-Phase Model for Solidification Transport Phenomena, Metallurgical Transactions B, vol. 22B, pp. 349-361.

Ohashi, O., Meguro, S., and Yamagata, T., 1996, Effect of Twist Angle on Mechanical Properties of Diffusion Bonded Joint Using Ni-Base Single Crystal Superalloy TMS-26, Mat. Trans. JIM, vol. 37, no. 9, pp. $1505-1510$

Patankar, S. V., 1980, Numerical Heat Transfer and Fluid Flow, Hemisphere Publishing Corporation.

Ruan, Z., Wang, S., and Zheng, Y., 1996, Microstructure and Bonding Behavior of a New Hf-Bearing Interlayer Alloy for Single Crystal Nickel-Base Superalloy, Scri. Mat., vol. 34, pp. 163-168.

Zheng, Y., Wang, R., and Li, C., 1987, Proceeding of the First ASM European and Techniques for structure Application, Paris, France, pp. 111-116. 

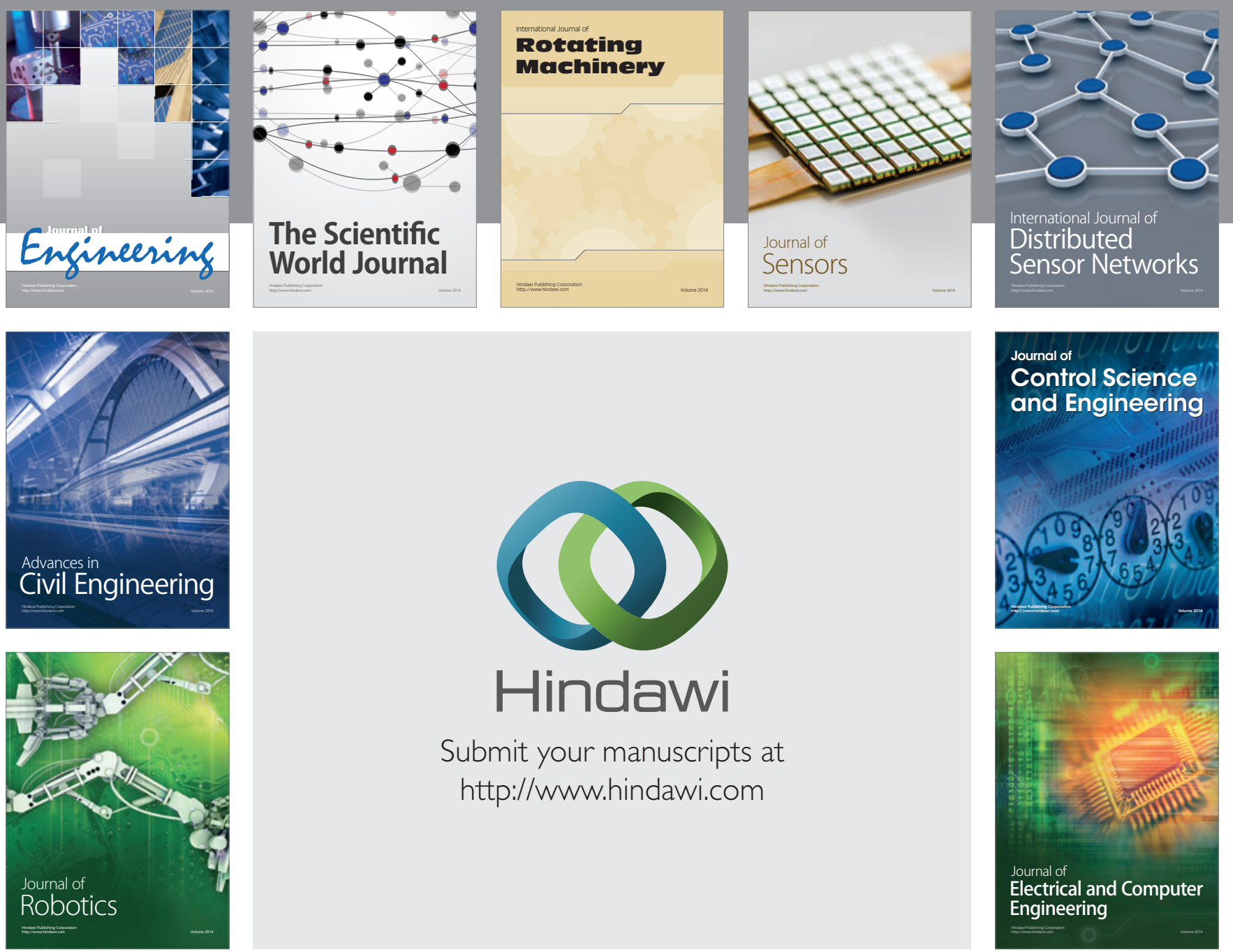

Submit your manuscripts at

http://www.hindawi.com
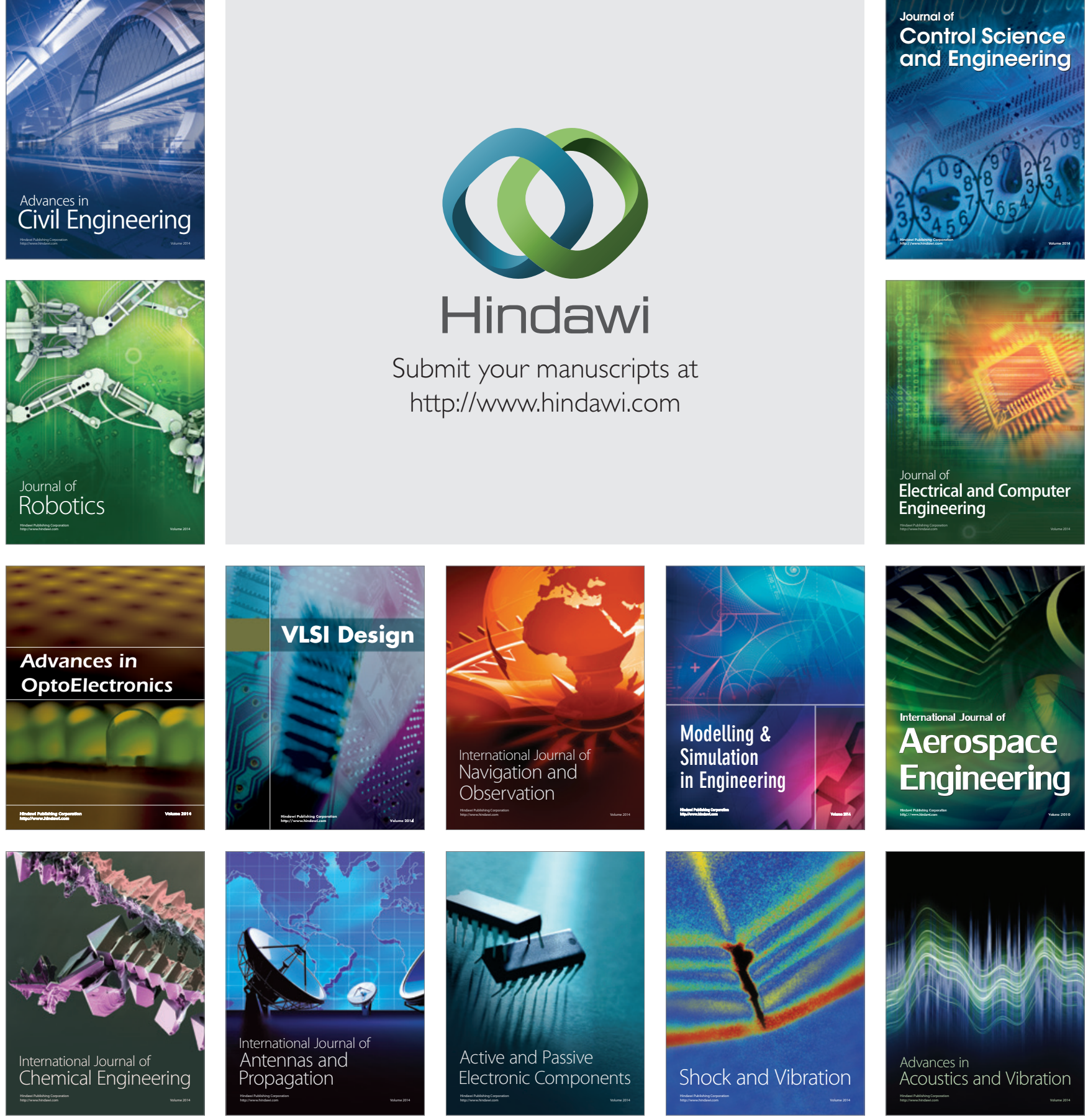